\title{
Etude préliminaire des relations entre le complexe majeur d'histocompatibilité (SLA) et des caractères de production chez le porc
}

\author{
P. CAPY * (1), Christine RENARD **, P. SELLIER * et M. VAIMAN ** \\ avec la collaboration technique de A. Derrien et J. Lecointre \\ * I.N.R.A., Station de Génétique quantitative et appliquée \\ ** Laboratoire de Radiobiologie appliquée, I.P.S.N.-D.P.R.-C.E.A. \\ Centre de Recherches zootechniques, $F-78350$ Jouy-en-Josas
}

\section{Résumé}

Des résultats préliminaires concernant les relations entre les gènes des locus SLA-A, SLA-B et SLA-C du complexe majeur d'histocompatibilité (SLA) du Porc et des caractères de production sont présentés. Cette étude porte sur 344 mâles et 195 femelles des races Large White, Landrace Français et Landrace Belge. Ces trois races diffèrent à la fois par la fréquence des gènes SLA étudiés et par la fréquence de certaines associations gamétiques (haplotypes). Un petit nombre d'associations significatives entre haplotypes SLA et performances de croissance et de carcasse sont mises en évidence : elles concernent en particulier l'haplotype 5.20.4, associé à de moins bonnes performances de croissance chez le Large White, et l'haplotype 1.15.18, associé à une adiposité accrue de la carcasse chez les Landrace Français et Belge.

Un complexe majeur d'histocompatibilité, appelé SLA (Swine Leucocyte Antigen), a été mis en évidence chez le Porc (Sus scrofa) par Vaman et al. (1970). Ce système présente une grande similitude de structure et probablement de fonction avec les autres complexes déjà connus, notamment ceux de l'Homme (HLA) et de la Souris (H2) (Levezrel, 1979). Dans ces deux dernières espèces, des associations entre le complexe majeur d'histocompatibilité et la prédisposition à certaines maladies ont été décelées (Dausset \& SvejgaARd, 1977). La recherche, chez les animaux domestiques, de relations entre les caractères antigéniques gouvernés par le complexe majeur d'histocompatibilité et les performances zootechniques, dont on sait qu'elles dépendent en partie de l'état de santé des individus, paraît intéressante. Ainsi, chez la poule, le complexe majeur d'histocompatibilité, qui est le système érythrocytaire $B$, influence un certain nombre de caractères d'importance économique, dont la résistance à la maladie de Marek (BriLes et al., 1977). L'objet de cette note est de présenter des résultats de caractère préliminaire concernant des antigènes du système SLA du porc.

(1) Adresse actuelle : Laboratoire de Biologie génétique et évolutive, C.N.R.S., F-91190 Gifsur-Yvette.

(2) Les commandes de tirés-à-part sont à adresser au Laboratoire de Radiobiologie appliquée. 
Sur le plan génétique, le système SLA se présente comme un ensemble de locus étroitement liés comportant deux régions principales (VAIMAN et al., 1979). La première région se compose de trois séries alléliques notées SLA-A, SLA-B, SLA-C, dont les produits antigéniques, dits de classe I et présents à la surface de toutes les cellules d'un individu, sont identifiés par des techniques sérologiques de lymphocytotoxicité décrites par VaIman et al. (1970). La deuxième région comporte des gènes notés $D / D R$ qui contrôlent respectivement la réaction lymphocytaire mixte et la synthèse des antigènes à expressions restreintes.

La présente étude concerne uniquement les antigènes de classe $I$. Les trois séries alléliques $\mathrm{A}, \mathrm{B}$ et $\mathrm{C}$ sont très polymorphes et comptent respectivement pour l'heure 9,7 et 5 spécificités contrôlées par des allèles codominants dont les fréquences varient d'une race à l'autre. L'étude de la transmission des antigènes SLA dans un grand nombre de familles non apparentées montre que les allèles aux différents locus présentent entre eux des associations gamétiques très importantes. Ceci a conduit à définir dans ces familles un nombre limité d'haplotypes standards (groupes d'allèles ségrégeant ensemble) que l'on peut légitimement utiliser pour la caractérisation du génotype SLA d'un individu, et cela avec un risque d'erreur faible (VAIMAN et al., 1979). Comme pour les allèles pris séparément, la fréquence de ces associations préférentielles d'allèles diffère d'une race à l'autre et certains haplotypes sont pratiquement spécifiques d'une race.

Les résultats présentés ici portent d'une part sur un échantillon de 334 mâles de race Large White $(\mathrm{N}=258)$ et Landrace Français $(\mathrm{N}=76)$ contrôlés dans trois stations de contrôle individuel de 1975 à 1979, d'autre part sur un échantillon de 195 femelles de race Large White $(\mathrm{N}=90)$, Landrace Belge $(\mathrm{N}=69)$ et Landrace Français $(\mathrm{N}=36)$ contrôlées dans une station de contrôle de descendance en 1979-1980. Ces animaux provenaient de plusieurs élevages de sélection des trois races concernées et la variété des origines permet de considérer comme bonne la représentativité des échantillons. Les performances zootechniques étudiées sont pour les mâles les trois caractères mesurés en station de contrôle individuel (gain moyen quotidien, indice de consommation et épaisseur de lard dorsal) ainsi qu'un indice de sélection combinant ces trois caractères et pour les femelles, les caractères d'engraissement et de carcasse classiquement mesurés dans les stations de contrôle de descendance (Ollivier et $a l ., 1981)$. Les échantillons sanguins sont prélevés par ponction veineuse et les caractéristiques SLA sont déterminées par le Laboratoire de Radiobiologie appliquée du C.E.A. à Jouy-en-Josas.

Nous avons retenu, pour cette étude, les haplotypes présentant une fréquence notable dans l'une au moins des races étudiées, soit 13 au total (pour plus de détails, voir CAPY, 1980). L'étude des fréquences de ces 13 haplotypes permet de caractériser les races étudiées par un ou plusieurs haplotypes. Ainsi, en regroupant les données relatives aux mâles et aux femelles, le Large White est caractérisé par l'haplotype 1.15.18 pour lequel la fréquence des porteurs (F) est égale à 0,30 et par l'haplotype 17 $(\mathrm{F}=0,17)$, le Landrace Français par les haplotypes $2.8 .11(\mathrm{~F}=0,32)$ et 16.11 $(\mathrm{F}=0,20)$ et le Landrace Belge également par les haplotypes $2.8 .11(\mathrm{~F}=0,62)$ et $16.11(\mathrm{~F}=0,33)$.

Les associations entre données immunologiques et données zootechniques ont été étudiées séparément dans chacun des 5 échantillons race-sexe. L'analyse a été faite en considérant, pour chaque échantillon race-sexe, les haplotypes observés à une 


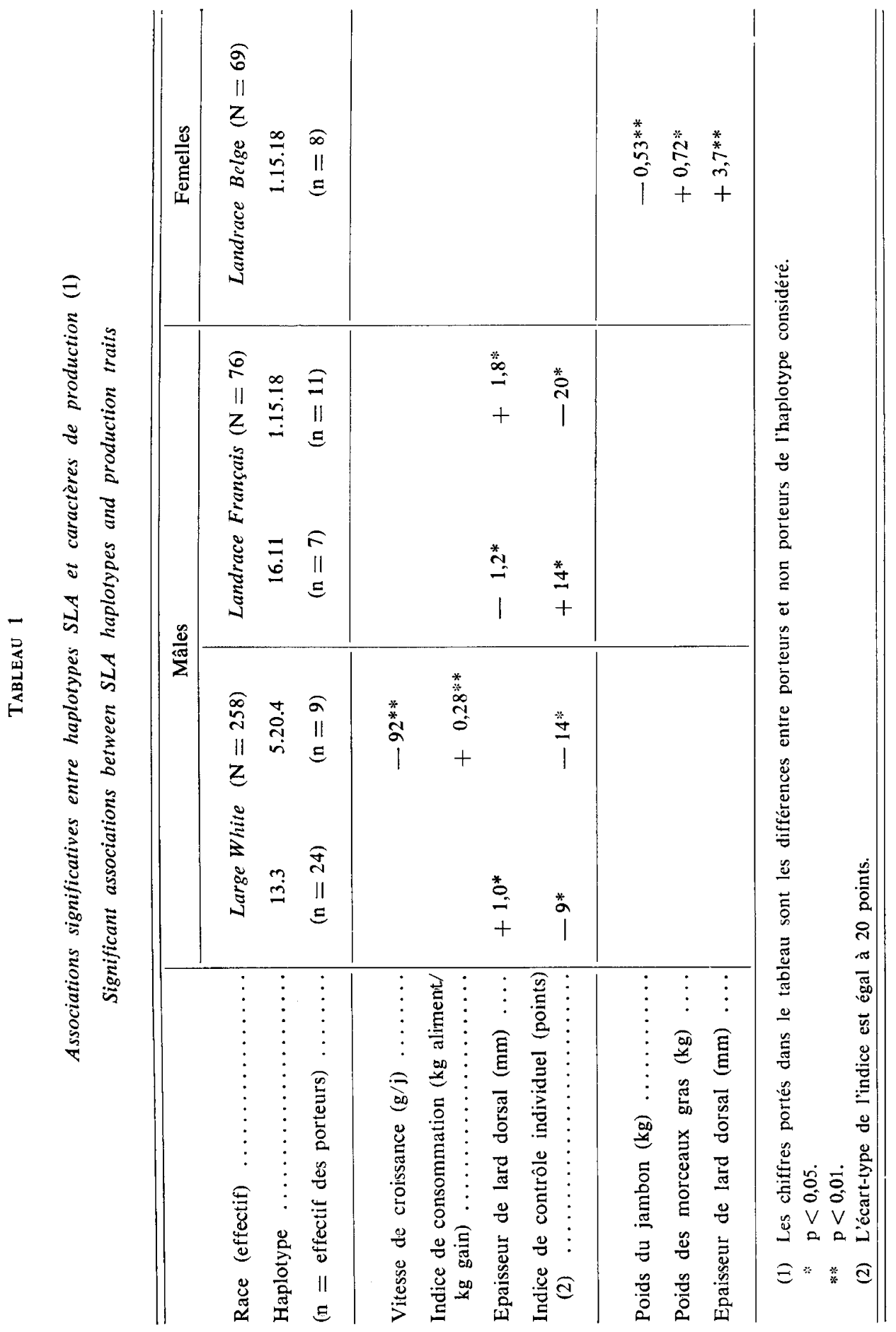


fréquence notable : les haplotypes retenus sont pris comme autant de facteurs à deux niveaux (présence ou absence) - en même temps que le facteur bande de contrôle pour l'analyse de variance des caractères zootechniques par la méthode des moindres carrés.

Nous rapportons dans le tableau I les résultats les plus significatifs de cette analyse : deux de ces résultats méritent une mention particulière. Il s'agit d'abord de l'association hautement significative entre l'haplotype 5.20.4 et la vitesse de croissance et l'indice de consommation chez les mâles Large White, les porteurs de cet haplotype ayant de moins bonnes performances d'engraissement que les non-porteurs. Cette association nous semble d'autant plus intéressante à souligner que la fréquence de l'haplotype 5.20.4 diminue au fil des années dans la race Large White (Varman \& RENARD, données non publiées) : ainsi cet haplotype n'a pas été retrouvé dans l'échantillon des femelles qui correspond aux données les plus récentes et ce fait n'a pas permis une éventuelle confirmation des résultats trouvés chez les mâles. La réduction de la fréquence de l'haplotype 5.20 .4 pourrait être une conséquence de la sélection pratiquée dans la race Large White sur les performances de croissance. D'autre part, une association significative entre l'haplotype 1.15 .18 et les caractères d'adiposité est trouvée tant chez les mâles Landrace Français que chez les femelles Landrace Belge, les animaux porteurs de cet haplotype étant plus gras que les non-porteurs. Rappelons que l'haplotype 1.15 .18 , relativement rare dans les deux populations Landrace étudiées ici, est à une fréquence particulièrement forte chez le Large White.

Ces observations ont un caractère tout à fait préliminaire, les effectifs d'animaux étudiés étant très limités. Elles demandent donc à être confirmées. Il faut noter aussi que le facteur père n'a pas été considéré dans l'analyse. Remarquons cependant que, sur les 9 porteurs de l'haplotype 5.20.4, deux seulement sont issus d'un même père, les sept autres n'ayant pas d'ancêtre commun sur deux générations. On peut aussi écarter l'hypothèse d'une association fortuite dans le cas de l'haplotype 1.15.18 qui a des effets similaires dans les deux types de Landrace. Ces premiers résultats sont à rapprocher de ceux de l'étude de JENSEN et al. (1968) qui concerne les associations entre groupes sanguins et caractères zootechniques. Parmi les 12 groupes sanguins étudiés dans la race Duroc, ce sont les groupes $\mathrm{C}$ et $\mathrm{J}$ qui sont le plus étroitement associés à des caractères de production comme les poids à 42 et 154 jours et l'épaisseur de lard dorsal. Or on sait que les systèmes $C$ et $J$ sont liés (la distance les séparant a été estimée à 5,7 $\pm 0,8$ centimorgans par MUIR \& RASMUSEN, 1974) et appartiennent au même groupe de liaison qe le complexe SLA, qui est distant d'environ 10 centimorgans du système $\mathbf{J}$ (HRUBAN et al., 1976). La signification commune des résultats de JENSEN et al. (1968) et de nos propres résultats mériterait d'être étudiée de façon plus approfondie.

Reçu pour publication en octobre 1981.

\section{Remerciements}

Nous tenons à remercier J. OWEN (U.P.R.A. porcine) et les directeurs des stations de sélection qui nous ont apporté leur concours pour cette étude. 


\author{
Summary \\ A preliminary study \\ of the relationships between the major histocompatibility complex (SLA) \\ and production traits in pigs
}

Preliminary results on the relationships between the SLA-A, SLA-B and SLA-C genes of the pig major histocompatibility complex (SLA) and production traits are presented. The study is based on 334 males and 195 females from the Large White, French Landrace and Belgian Landrace breeds. These three breeds differ both by the frequency of the SLA genes under study and by the frequency of some gametic associations between them (haplotypes). A small number of significant associations between SLA haplotypes and growth and carcass traits are found : they particularly involve the haplotype 5.20.4, associated with a lower growth performance in the Large White breed, and the haplotype 1.15.18, associated with a higher carcass fatness in the French and Belgian Landrace breeds.

\title{
Références bibliographiques
}

Briles W.E., Stone H.A., Cole R.K., 1977. Marek's disease : effects of B histocompatibility in resistant and susceptible chicken lines. Science, 195, 193-195.

CAPY P., 1980. Etude des relations entre le complexe majeur d'histocompatibilité (SLA) et quelques caractères de production chez le porc. D.E.A. de Génétique quantitative et appliquée, Université de Paris XI.

Dausset J., Svejgaard A., 1977. HLA and Diseases. Munksgaard, Copenhagen.

Hruban V., Simon M., Hradecky J., Jilek F., 1976. Linkage of the pig main histocompatibility complex and the J blood group system. Tissue Antigens, 7, 267-271.

Jensen E.L., Smith C., Baker L.N., Cox D.F., 1968. Quantitative studies on blood group and serum protein systems in pigs. II - Effects on production and reproduction. J. anim. Sci., 27, 856-862.

Leveziel H., 1979. Le complexe d'histocompatibilité majeur chez l'homme et chez les animaux. Ann. Génét. Sél. anim., 11, 281-356.

Muir W.M., RASMUSEN B.A., 1974. A combined estimate of recombination between the genes for $\mathrm{C}$ and $\mathrm{J}$ blood groups in pigs. Anim. Blood Grps biochem. Genet., 5, 133-135.

Ollivier L., Derrien A., Molenat M., 1981. Paramètres génétiques des femelles Large White et Landrace Français du contrôle de descendance sur la période 1970-1980. In : $13^{c s}$ Journées de la Recherche porcine en France, 293-298, Institut technique du Porc, Paris.

Vaiman M., Chardon P., Renard C., 1979. Genetic organization of the pig SLA complex. Studies on nine recombinants and biochemical and lysostrip analysis. Immunogenetics, 9, 353-361.

Vaiman M., Renard C., Lafage P., Ameteau J., Nizza P., 1970. Evidence for a histocompatibility system in pig. Transplantation, 10, 155-164. 